Steiniger Weg zu fairen Preisen im Güterverkehr

\title{
Brüssel auf der Bremse
}

\section{Die Ausweisung aller verursachten Kosten durch LKW-Verkehr ist seit langem auf der politischen Agenda der Europäischen Union. Eine genauere Betrachtung der entsprechenden Richtlinie macht aber deutlich, dass die vorgeschriebenen Mindestanforderungen keineswegs das gesamte mögliche Instrumentarium zur Internalisierung von Umweltkosten zwingend machen.}

$\mathrm{F}$ Von Markus Liechti aire und effiziente Preise im Verkehr werden seit über einer Dekade auf nationaler und europäischer Ebene diskutiert. Mit dem ungebrochenen Wachstum im Verkehrsbereich wachsen auch die verkehrsbedingten Probleme für Mensch und Umwelt. Experten sind sich während der vergangenen zehn Jahre zunehmend einig geworden, dass diese Probleme durch falsche Preissignale verursacht werden. Die Verkehrspreise sind zu tief und somit wird zu viel Verkehr verursacht und entstehen für die Gesellschaft und die öffentliche Hand zu hohe Kosten. Diese Kosten werden als ,externe Kosten' bezeichnet, da der Verkehrsbereich dafür nicht selbst aufkommt. Daraus entsteht ein unfairer Wettbewerbsvorteil für jene Verkehrsträger, die die größten Schäden und Kosten verursachen.

Die europäischen Institutionen in Brüssel haben ebenfalls erkannt, dass es eine europäische Regelung für die Erhebung von Verkehrsgebühren geben sollte. Wiederholt verlangten sowohl Europäische Kommission als auch Rat in Form ihres Weißbuches und im Rahmen der Gipfeltreffen der Staatsoberhäupter 2001 in Götheborg und 2002 in Barcelona, dass die Verkehrsträger die von ihnen verursachten Kosten übernehmen müssen (1).

\section{Knackpunkt Gebühren}

Die Europäische Kommission legte im Sommer 2003 einen Vorschlag zur Revision der Eurovignettenrichtlinie vor. Die derzeitige Richtlinie 1999/62 (2) erlaubt die Erhebung von Gebühren für den Schwerverkehr ab zwölf Tonnen Gesamtgewicht. Diese Gebühren können einerseits in Form einer zeitabhängigen Gebühr, was der traditionellen Eurovignette entspricht, erhoben werden. Andererseits erlaubt sie auch die Erhebung von distanzabhängigen Gebühren, wie sie in Österreich seit Beginn dieses Jahres erhoben werden und in der Schweiz seit 2001 bestehen. Die geplante LKW-Maut in Deutschland entspricht ebenfalls diesem Ansatz.
Der Kommissionsvorschlag geht nur unwesentlich darüber hinaus, was schon mit der bestehenden Richtlinie möglich ist. Die Änderungen betreffen vorwiegend die Möglichkeit zur Erhebung von Zuschlägen in sensiblen Gebieten und die Variabilisierung aufgrund von Umweltwirkungen. Der Vorschlag der Kommission ist allerdings eher enttäuschend, da dieser weit hinter dem zurückblieb, was der Gipfel von Barcelona oder selbst die Kommission in ihrem Weißbuches im Jahre 2001 forderten. Dieser Vorschlag weist insbesondere die folgenden Mängel auf:

- Preis und Finanzierungsinstrumente werden vermischt: Preisinstrumente im Verkehr sollten Anreize zum effizienten Umgang mit Verkehrsinfrastruktur liefern. Dieses Ziel sollte nicht vermischt werden mit dem Ziel, Mittel für Investitionen in Verkehrsprojekte zu generieren. Genau diese beiden Ziele werden mit dem Kommissionsvorschlag verfolgt, der die Mitgliedsstaaten verpflichtet, die Mittel Zweck gebunden für den Verkehrssektor zu verwenden. Es ist wenig wahrscheinlich, dass damit die eingenommenen Mittel am zweckmäßigsten verwendet werden und den größten Nutzen für Wirtschaft und Gesellschaft abwerfen. Schließlich ist der Zwang zur Zweckbindung eine Einmischung der EU in die Budgethoheit der Mitgliedsstaaten.

- Der Anwendungsbereich der Richtlinie ist nicht umfassend genug, da er auf das Transeuropäische Netz beschränkt ist und die Mitgliedsländer die Europäische Kommission anfragen müssen, um auf die Gebühren auf Parallelstraßen ausdehnen zu können. Dies widerspricht dem Verursacherprinzip, da auf allen Straßen, nicht nur dem Transeuropäischen Straßennetz, Kosten anfallen, die von den Benutzern getragen werden sollten. Zudem widerspricht dies ebenfalls der Autonomie der Mitgliedsländer, auf diesen Straßen ebenso wie auf dem untergeordneten Netz, was durch den Kommissionsvorschlag erlaubt ist, nichtdiskriminierende Gebühren erheben zu können.
- Das Verusacherprinzip wird nur unvollständig angewandt, da die externen Umwelt- und Staukosten nur unvollständig oder gar nicht berücksichtigt werden. Neben den Infrastrukturkosten, die auch Baukosten der vergangenen 15 Jahre enthalten dürfen, können nur die ungedeckten Unfallkosten und direkt anfallende Kosten zur Lärmreduktion berücksichtigt werden. Dadurch gibt es weiterhin einen Wettbewerbsnachteil für Verkehrsträger, die weniger Umweltkosten verursachen, wie zum Beispiel Eisenbahnen und Schiffe.

Neben diesen drei Mängeln bestehen weitere Widersprüche im Kommissionsvorschlag. T\&E hat von einem anerkannten Ökonomen einen Alternativvorschlag erarbeiten lassen, der eine konsistente Revision der Eurovignettenrichtlinie und die vollständige Anwendung des Verursacherprinzips zulassen würde (3).

\section{Ausblick}

Die Mehrheit dieser Anliegen wurde vom Europäischen Parlament berïcksichtigt und in den Bericht integriert, der in der Plenumssitzung vom 20. April in Straßburg verabschiedet wurde. Im Verkehrsministerrat vom 9. März konnte hingegen keine Einigung gefunden werden. Die Europäische Kommission hat auf der Zweckbindung beharrt und erwartungsgemäß den Widerspruch der Mitgliedsländer hervorgerufen. Damit die Änderung der Richtlinie angenommen wird, braucht es die Zustimmung von Verkehrsministerrat und Europäischem Parlament. Wegen des Scheiterns im Rat ist es fraglich, ob eine Einigung möglichst rasch erzielt werden kann, obwohl das Resultat im Europäischen Parlament eine gute Voraussetzung zur Einigung bilden würde, da die Mittelverwendung den Mitgliedsstaaten überlassen wird.

\section{Anmerkungen}

(1) Weißbuch der Europäischen Kommission: Europäische Verkehrspolitik 2010. Brüssel 2001.

(2) Richtlinie 1999/62 für die Erhebung von Gebühren für die Benutzung bestimmter Verkehrswege durch schwere Nutzfahrzuge.

(3) Kågeson, Per: Amending the Eurovignette Directive An alternative to the European Commission's proposal. T\&E 03/4, Brüssel 2004.

\section{Der Autor}

Dr. Markus Liechti ist Projektleiter beim Europäischen Verband für Verkehr und Umwelt in Brüssel (T\&E). Kontakt: T\&E, 34 Boulevard de Waterloo, B-1000 Brüssel. Tel. 0032-2-5029909, E-Mail: markus.liechti@t-e.nu 
(c) 20I0 Authors; licensee IÖW and oekom verlag. This is an article distributed under the terms of the Creative Commons Attribution Non-Commercial No Derivates License (http://creativecommons.org/licenses/by-nc-nd/3.o/), which permits unrestricted use, distribution, and reproduction in any medium, provided the original work is properly cited. 